\title{
The Association of Serum 25-Hydroxyvitamin D and Carcinoembryonic Antigen in Locally Advanced Colorectal Cancer With Neoadjuvant Chemoradiotherapy
}

\section{Jing Shi}

Changzhou Cancer Hospital: Changzhou City Fourth People's Hospital

\section{Yongqi Zhang}

Changzhou Cancer Hospital: Changzhou City Fourth People's Hospital

\section{Xiang He}

Changzhou Cancer Hospital: Changzhou City Fourth People's Hospital

\section{Hong Ye}

Changzhou Cancer Hospital: Changzhou City Fourth People's Hospital

\section{Liping Cheng}

Changzhou Cancer Hospital: Changzhou City Fourth People's Hospital

\section{Chunying Jiang}

Changzhou Cancer Hospital: Changzhou City Fourth People's Hospital

\section{Haiyang Zhong}

Changzhou Cancer Hospital: Changzhou City Fourth People's Hospital

\section{Litao Yu ( $\square$ yulitaoylt@163.com)}

Changzhou Cancer Hospital: Changzhou City Fourth People's Hospital https://orcid.org/0000-00016436-9753

\section{Research}

Keywords: 25-hydroxyvitamin D, carcinoembryonic antigen, neoadjuvant chemoradiotherapy(nCRT), locally advanced colorectal cancer(LARC)

Posted Date: February 8th, 2021

DOI: https://doi.org/10.21203/rs.3.rs-165466/v1

License: (a) (i) This work is licensed under a Creative Commons Attribution 4.0 International License. Read Full License 


\section{Abstract}

\section{Backgrond}

We aimed to describe 25-hydroxyvitamin $D[25(\mathrm{OH}) \mathrm{D}]$ and carcinoembryonic antigen association levels in patients with locally advanced colorectal cancer (LARC) treated with neoadjuvant chemoradiotherapy (nCRT).

Methods

Between July 2012 and September 2018, the records of 92 stage II/III colorectal cancer patients with treated with nCRT followed by radical surgery were reviewed retrospectively. Enzyme-linked immunosorbent assay (ELISA) and chemiluminescence immunoassay was used to analyze the patient's carcinoembryonic antigen (CEA) and 25(OH) D concentrations in serum.

Results

The serum levels of $25(\mathrm{OH}) \mathrm{D}$ in patients were significantly lower than healthy individuals, just like CEA levels were significantly higher than healthy individuals. The serum CEA in pre-nCRT was significantly lower than in post-nCRT patients, while the serum 25(OH) D from pre-nCRT patients was significantly higher than post-nCRT patients. Our results showed that the pre-nCRT CEA and 25(OH) D level is a risk factor of LARC.

Conclusions

Neoadjuvant chemoradiotherapy induces a fall in circulating

25(OH) D and carcinoembryonic antigen (CEA). Plasma $25(\mathrm{OH}) \mathrm{D}$ levels is a prognostic biomarker with low 25(OH) D associated with poorer survival. Thus, serum 25(OH) D and CEA levels might be of value in evaluating the pathogenesis and risk of LARC in the future. Moreover, serum CEA or 25(OH) D levels were associated with patient's clinical and pathological features providing data for risk and prognostic prediction.

\section{Background}

Colorectal cancer (CRC) is a common type of cancer worldwide [1]. In China, the estimated new cases and deaths of CRC were 376300 and191000, respectively, in 2015[2]. Neoadjuvant chemoradiotherapy (nCRT) has been used as a consensus-based treatment modality for stage II/III colorectal cancer [3]. The move has heightened sphincter preservation rates and lower local recurrence rates, and also down staging of the disease. Accumulating evidence indicates that high levels of circulating vitamin $D$ are an anti-cancer factor, which may reduce the risk of CRC [4-6]. 
In the human body, vitamin D participates in the metabolism of calcium, phosphorus and other substances, and it is believed to be highly associated with cancer as well as other chronic diseases[7]. Accumulating data suggest that vitamin D deficiency is highly prevalent among patients with CRC. In the liver, vitamin $D$ is converted by 25-hydroxylase into 25 hydroxyl vitamin D3 (25(OH)D3), the main circulating form of vitamin $\mathrm{D}$ and the most reliable measurement of an individual's vitamin $\mathrm{D}$ status[8].

The neoadjuvant chemo radiotherapy resistance contributes to substantial mortality in advanced colorectal cancer, it would be representatives for alternative or rigorous treatment approaches. So, identifying predictive biomarkers of response in advanced following nCRT would be of significant clinical relevance. Among the biomarkers, Carcinoembryonic antigen (CEA) is the most useful predictor to estimate the sensitivity of nCRT in colorectal cancer [9]. So far, the expression levels of 25(OH) D and CEA in locally advanced colorectal cancer, and their association with advanced colorectal cancer pathogenesis and risk are still ambiguous. In this study, we analyzed the serum 25(OH) D and CEA levels in pre-and post-neoadjuvant chemo radiotherapy locally advanced colorectal cancer patients and correlated them with clinical features and the risk of locally advanced colorectal cancer.

\section{Materials And Methods 2.1 Patients}

Ninety two patients diagnosed with locally advanced colorectal cancer were enrolled consecutively in this study in a period of time from July 2012 and September 2018. The diagnosis was made by pathologic examination. During hospitalization; all patients had no outdoor activity and were provided diet without calcium or vitamin D supplements. 75 healthy individuals, aging from 45 to 77 years old, who had regular diet without calcium or vitamin $D$ supplements before blood drawing, were selected as controls with matched ages and genders to the patient group. The study was approved by the Institutional Review Board of the Changzhou Cancer Hospital Autonomous Region for the use of human materials.

\subsection{Blood sample collection}

Fasting blood samples were obtained either the day before nCRT or other treatments (baseline) and on day 14 after $\mathrm{nCRT}$, respectively, using the serum separation tube (BD healthcare). All patients with locally advanced colorectal cancer had paired pre-nCRT and post-nCRT blood samples. The blood samples were transported to the Department of Laboratory Medicine within 30 minutes of collection. The samples were then centrifuged at $1500 \mathrm{~g}$ at $4^{\circ} \mathrm{C}$ for 10 minutes; the serum was collected into a $2 \mathrm{~mL}$ of centrifuge tube and stored at $-80^{\circ} \mathrm{C}$ until analysis.

\subsection{Enzyme-linked immunosorbent assay}

CEA was measured by using the Quantizing ELISA Human CEA Immunoassay kit from R \& D Systems, in following the manufacturer's instructions. In brief, $100 \mu \mathrm{L}$ of Assay Diluent RD1-19 was added to each well in the microplate; then, $50 \mu \mathrm{L}$ of standard, control, or sample were added per well and covered with the adhesive strip to incubate for 1 hour at room temperature on a horizontal orbital microplate shaker; all 
the liquid was aspirated and washed by adding $400 \mu \mathrm{L}$ Wash Buffer for 4 times; after the last wash, inverted and blotted against the plate through clean paper towels to drain he remaining wash buffer; added $200 \mu \mathrm{L}$ of Human CEA Conjugate to each well and covered with a new adhesive strip to incubate for 2 hours at room temperature on the shaker; repeated the aspiration and wash; then added $200 \mu \mathrm{L}$ of Substrate Solution to each well to incubate for 30 minutes at room temperature on the benchtop by protecting from light; following this step, $50 \mu \mathrm{L}$ of stop solution was added to stop the color development; and finally, measured the plate immediately on a microplate reader at OD $450 \mathrm{~nm}$.

\subsection{Chemiluminescence immunoassay}

In clinical laboratory, chemiluminescence immunoassay is widely used to measure serum vitamin D (VD) because of its automatic capability with clinical satisfaction. In this study, serum VD was quantitatively analyzed on a Roche cobas E601 electrochemiluminescence immunoassay platform using a Roche cobas Elecsys Vitamin D total (25Hydroxyvitamin D, REF 05894913 190, Roche Diagnostics GmbH) assay kit.

\subsection{Statistical analysis}

IBM SPSS Statistical 24.0 (SPSS Inc Chicago) was performed for statistical analysis. Differences in basic characteristics of patients were evaluated by using independent samples $t$ test. Paired samples $T$ test was performed to compare the difference in each parameter of paired pre- and post- nCRT serums or $25(\mathrm{OH}) \mathrm{D}$ levels. Moreover, Pearson's correlation analysis was applied to evaluate the association between serum CEA and 25(OH) D levels in LARC. The pre- and post- $n C R T$ 25(OH)D and pre- and postnCRT CEA ( $\geq$ median and $<$ median) levels were stratified by using conditional logistic regression (multivariate analysis) to evaluate whether serum CEA or 25(OH)D is a risk factor for LARC, which estimated odds ratios (OR) and $95 \%$ confidence intervals (CI). A P-value of $<0.05$ was considered as statistically significant. Serum $25(\mathrm{OH}) \mathrm{D}$ and CEA levels were correlated with clinical and pathological features including age, gender, lesion site, tumor size, lymph node involvement,CRT regimen.

\subsection{Ethics statement}

The study was approved by the Institutional Review Board of the

Changzhou Cancer Hospital Autonomous Region for the use of human materials.

\section{Results}

\subsection{Correlation of serum CEA and 25(OH) D levels with clinical-pathological features in LARC patients}

The correlation of serum CEA and 25(OH) D levels with clinical pathological features from 92 patients with LARC were summarized as shown in Table 1. Of 92 patients with LARC, there were 39 females and 
53 males with ages ranging from 35 to 82 years (a median age of 61 years). In this study, colon was the main lesion site of the tumor (49/92).

According to the parameters we collected, we divided each parameter into two groups, which was analyzed through univariate analysis (displayed in Table 1). In independent samples test, our data showed that the groups, tumor size $₫ 5.0 \mathrm{~cm}$, lymph node involvement, that has no distinctly lower serum post-nCRT CEA levels than the groups(pख0.05). As for the levels of pre-nCRT serum 25(OH) D, males, tumor size $<5.0 \mathrm{~cm}$, lymph node invasion groups were significantly higher $(P=0.005, P=0.008, P=0$ .049 , respectively). While with post-nCRT $25(\mathrm{OH}) \mathrm{D}$ levels, males, lymph node invasion, and tumor size < $5.0 \mathrm{~cm}$ had significantly higher levels ( $p<0.05$, respectively). In paired samples test, the post-nCRT CEA concentration of patients were significantly lower than the pre-nCRT CEA levels except the group of lymph node invasion. Most surprisingly, in the standard radiotherapy and chemotherapy regimens, Capecitabine did not change the $25(\mathrm{OH}) \mathrm{D}$ levels before and after neoadjuvant chemo radiotherapy. 
Table 1

Correlation of serum CEA and 25(OH) D with clinical-pathological features

\begin{tabular}{|c|c|c|c|c|c|c|c|}
\hline \multirow[t]{2}{*}{ Parammeters } & \multirow{2}{*}{$\begin{array}{l}\text { Toatal } \\
(\mathrm{n}= \\
92)\end{array}$} & \multicolumn{3}{|c|}{$\begin{array}{l}\text { CEA } \\
(X \pm S \mathrm{mmol} / \mathrm{l})\end{array}$} & \multicolumn{3}{|c|}{$\begin{array}{l}\text { 25(OH)D } \\
(\mathrm{X} \pm \mathrm{S} \mathrm{nmol} / \mathrm{l})\end{array}$} \\
\hline & & prenCRT & post-nCRT & $P^{b}$ & pre-nCRT & $\begin{array}{l}\text { post- } \\
\text { nCRT }\end{array}$ & $\mathrm{P}^{\mathrm{b}}$ \\
\hline \multicolumn{8}{|l|}{$\operatorname{Age}(\mathrm{Y})$} \\
\hline$\geq 60$ & 45 & $24.56 \pm 12.7$ & $20.13 \pm 10.4$ & $0.036 *$ & $52.11 \pm 4.2$ & $49.89 \pm 7.1$ & $0.037 *$ \\
\hline$<60$ & 47 & $28.56 \pm 11.7$ & $22.14 \pm 9.7$ & $0.024 *$ & $51.37 \pm 3.3$ & $48.92 \pm 7.1$ & $0.017 *$ \\
\hline$p^{a}$ & & 0.059 & 0.170 & & 0.174 & 0.257 & \\
\hline \multicolumn{8}{|l|}{ Gender } \\
\hline Female & 39 & $27.35 \pm 1.9$ & $25.89 \pm 2.4$ & $0.001 *$ & $43.62 \pm 3.7$ & $40.93 \pm 6.4$ & $0.012 *$ \\
\hline male & 53 & $30.13 \pm 6.3$ & $27.94 \pm 5.4$ & $0.028 *$ & $46.13 \pm 4.5$ & $43.35 \pm 7.1$ & $0.008 *$ \\
\hline$P^{a}$ & & $0.014 *$ & $0.008 *$ & & $0.005 *$ & $0.048 *$ & \\
\hline \multicolumn{8}{|l|}{ Lesion site } \\
\hline Colon & 49 & $32.17 \pm 5.8$ & $28.87 \pm 6.3$ & $0.004 *$ & $45.31 \pm 3.7$ & $43.26 \pm 7.7$ & $0.048 *$ \\
\hline Rectum & 43 & $30.52 \pm 4.7$ & $27.69 \pm 5.1$ & $0.004 *$ & $44.34 \pm 6.1$ & $41.92 \pm 5.5$ & $0.028 *$ \\
\hline $\mathrm{P}^{\mathrm{a}}$ & & 0.070 & 0.162 & & 0.183 & 0.167 & \\
\hline \multicolumn{8}{|l|}{ Tumor size } \\
\hline$\geq 5.0 \mathrm{~cm}$ & 42 & $55 . .69 \pm 6.5$ & $53.94 \pm 5.8$ & 0.098 & $49.32 \pm 5.5$ & $46.17 \pm 4.3$ & $0.002 *$ \\
\hline$<5.0 \mathrm{~cm}$ & 50 & $51.33 \pm 7.6$ & $48.69 \pm 5.9$ & $0.027 *$ & $52.76 \pm 8.1$ & $49.86 \pm 5.9$ & $0.013 *$ \\
\hline$P^{a}$ & & $0.002 *$ & $0.001 *$ & & $0.008 *$ & $0.0004 *$ & \\
\hline \multicolumn{8}{|l|}{$\begin{array}{l}\text { Lymph node } \\
\text { invasion }\end{array}$} \\
\hline YES & 38 & $69.33 \pm 8.6$ & $68.13 \pm 7.3$ & 0.257 & $53.12 \pm 4.6$ & $50.33 \pm 5.2$ & $0.007 *$ \\
\hline NO & 54 & $68.01 \pm 9.6$ & $65.43 \pm 5.1$ & $0.042 *$ & $51.09 \pm 7.1$ & $48.12 \pm 7.5$ & $0.018 *$ \\
\hline
\end{tabular}

Note: $P^{a}$ represents the comparison of intra-parameter. $P^{r}$ epresents the comparison of CEA and 25(OH) D in pre- and post- $n C R T$ LARC.

Abbreviations: Post-nCRT, post-neoadjuvant chemoradiotherapy; Pre-nCRT, pre-neoadjuvant chemoradiotherapy.

*Means $P<0.05$ 


\begin{tabular}{|c|c|c|c|c|c|c|c|}
\hline \multirow[t]{2}{*}{ Parammeters } & Toatal & \multicolumn{2}{|c|}{$\begin{array}{l}\text { CEA } \\
(X \pm S \mathrm{mmol} / \mathrm{l})\end{array}$} & \multicolumn{3}{|c|}{$\begin{array}{l}\text { 25(OH)D } \\
(\mathrm{X} \pm \mathrm{S} \mathrm{nmol} / \mathrm{l})\end{array}$} & \multirow[b]{2}{*}{$\mathrm{P}^{\mathrm{b}}$} \\
\hline & $\begin{array}{l}(n= \\
92)\end{array}$ & prenCRT & post-nCRT & $P^{b}$ & prenCRT & $\begin{array}{l}\text { post- } \\
\text { nCRT }\end{array}$ & \\
\hline $\mathrm{P}^{\mathrm{a}}$ & & 0.250 & $0.026 *$ & & $0.049 *$ & $0.049 *$ & \\
\hline \multicolumn{8}{|l|}{ CRT regimen } \\
\hline Capecitabine & 40 & $45.20 \pm 5.6$ & $41.81 \pm 6.2$ & $0.006 *$ & $58.24 \pm 9.2$ & $56.21 \pm 5.2$ & 0.114 \\
\hline FOLFOX & 52 & $46.22 \pm 4.5$ & $43.51 \pm 4.3$ & $0.001 *$ & $57.81 \pm 8.6$ & $54.85 \pm 7.7$ & $0.033 *$ \\
\hline $\mathrm{p}^{\mathrm{a}}$ & & 0.167 & 0.071 & & 0.409 & 0.157 & \\
\hline
\end{tabular}

Note: $P^{a}$ represents the comparison of intra-parameter. $P^{r}$ epresents the comparison of CEA and 25(OH) D in pre- and post- $n C R T$ LARC.

Abbreviations: Post-nCRT, post-neoadjuvant chemoradiotherapy; Pre-nCRT, pre-neoadjuvant chemoradiotherapy.

*Means $P<0.05$

\subsection{Serum CEA and 25(OH) D levels in pre-and post-nCRT LARC patients}

Table 2 shows that the serum CEA in pre- and post-nCRT LARC patients was significantly higher than the controls ( $\mathrm{P}<0.001$ for both). While serum 25(OH) D in both pre- and post-nCRT LARC patients were significantly lower than the controls ( $P<0.001$ for both). Moreover, the CEA levels in pre-nCRT LARC patients was significantly lower than the post- LARC levels $(P<0.005)$. However, the level of pre-nCRT 25(OH) D was significantly higher than post-nCRT 25(OH) $D(P<0.005)$. 
Table 2

Serum CEA and 25(OH) D levels in pre-and post- neoadjuvant chemoradiotherapy LARC patients and controls

\begin{tabular}{|c|c|c|c|c|c|c|}
\hline \multirow[t]{3}{*}{ Number } & \multirow{2}{*}{\multicolumn{3}{|c|}{$\begin{array}{l}\text { CEA } \\
(X \pm S \mathrm{mmol} / \mathrm{l})\end{array}$}} & \multirow{2}{*}{\multicolumn{3}{|c|}{$\begin{array}{l}25(\mathrm{OH}) \mathrm{D} \\
(\mathrm{X} \pm \mathrm{S} \mathrm{nmol} / \mathrm{l})\end{array}$}} \\
\hline & & & & & & \\
\hline & pre-nCRT & post-nCRT & P2 & pre-nCRT & post-nCRT & P2 \\
\hline Cases $(n=92)$ & $44.56 \pm 10.2$ & $30.56 \pm 10.2$ & $<0.005 *$ & $51.51 \pm 3.2$ & $42.11 \pm 5.2$ & $<0.005 *$ \\
\hline controls $(n=75)$ & $1.56 \pm 2.7$ & & & $77.58 \pm 12.2$ & & \\
\hline P1 & $<0.001 *$ & $<0.001 *$ & & $<0.001 *$ & $<0.001 *$ & \\
\hline \multicolumn{7}{|c|}{$\begin{array}{l}\text { Note: } P 1 \text { represents the difference between patients and controls. } P 2 \text { represents the difference } \\
\text { between pre-neoadjuvant chemoradiotherapy CEA or } 25(\mathrm{OH}) D \text { and post-neoadjuvant } \\
\text { chemoradiotherapy CEA or } 25(\mathrm{OH}) D \text {. }\end{array}$} \\
\hline \multicolumn{7}{|c|}{$\begin{array}{l}\text { Abbreviations: Post-nCRT, post-neoadjuvant chemoradiotherapy; Pre-nCRT pre-neoadjuvant } \\
\text { chemoradiotherapy }\end{array}$} \\
\hline \multicolumn{7}{|l|}{${ }^{*}$ Means $P<0.05$} \\
\hline
\end{tabular}

\subsection{Serum CEA and 25(OH) D levels and the risk of LARC}

So as to understand whether serum CEA and $25(\mathrm{OH}) \mathrm{D}$ is a risk factor for LARC, multivariate analysis and conditional logistic regression were performed and the results are displayed in Table 3 . The results showed that the pre-nCRT CEA level is a risk factor for $L A R C(O R=13.145, \mathrm{Cl}=1.71-110.31, \mathrm{P}=0.012)$, while the post-nCRT $25(\mathrm{OH}) \mathrm{D}$ level is a protective factor $(\mathrm{OR}=0.083, \mathrm{Cl}=0.01-0.84, \mathrm{P}=0.040)$.

Table 3

Evaluation of pre- and nCRT serum CEA and 25(OH) D as a risk factor in LARC patients

\begin{tabular}{|c|c|c|c|c|c|c|}
\hline \multirow[t]{2}{*}{ Factor } & & Regression & OR & $\mathrm{Cl}$ & Walad $\chi^{2}$ & p \\
\hline & & \multicolumn{5}{|l|}{ Coefficient B } \\
\hline \multirow[t]{2}{*}{ CEA } & Pre-nCRT & 2.385 & 13.145 & $1.71-110.31$ & 6.151 & $0.012 *$ \\
\hline & Post-nCRT & 10.331 & 37908.621 & $0.00-1.35 E+31$ & 0.072 & 0.768 \\
\hline \multirow[t]{2}{*}{$25(\mathrm{OH}) \mathrm{D}$} & Pre-nCRT & 1.693 & 5.376 & $0.53-43.31$ & 2.371 & 0.132 \\
\hline & Post-nCRT & -2.334 & 0.083 & $0.01-0.84$ & 4.331 & $0.040 *$ \\
\hline \multicolumn{7}{|c|}{$\begin{array}{l}\text { Abbreviations: } C l, 95 \% \text { confidence intervals; OR, odds ratios; Post- } n C R T \text { post- neoadjuvant } \\
\text { chemoradiotherapy; Pre-nCRT pre- neoadjuvant chemoradiotherapy }\end{array}$} \\
\hline
\end{tabular}

\subsection{Correlation between serum CEA and 25(OH) D levels}


Our research results demonstrated that there was a weak correlation between both pre-nCRT CEA and post-nCRT CEA with 25(OH) D levels (Table 4, Pearson - .126 and .154, $P=.265$ and .171, respectively).

Table 4

Comparison between serum CEA and 25(OH) D in LARC by Pearson's correlation analysis

\begin{tabular}{|lll|}
\hline Paired & PrenCRT CEA VS 25(OH)D & Post-nCRT CEA VS 25(OH)D \\
\hline Pearson correlation & -.126 & .154 \\
\hline P-value & .265 & .171 \\
\hline
\end{tabular}

\section{Discussion}

Several studies have predicted the sensitivity of $\mathrm{nCRT}$ in colorectal cancer. Imaging modalities that were used for evaluation included CT and ERUS[10]. The frequency of vitamin D insufficiency in patients with locally advanced CRC has not been reported in larger studies. Several studies suggested that the expression levels of four biomarkers ( $p 53, \mathrm{VEGF}, \mathrm{p} 21$, and Ki67) were significantly correlated with PCR to nCRT (96.3\%). However, this scoring system showed only $46.3 \%$ specificity. Her-2, pre-CEA, and post-CEA were also considered as predictors and of their disadvantages $[11,12]$. Altered CEA was proved more significant to reflect the sensitivity of colon cancer to chemotherapy than pre- or post-CEA [13, 14]. So, $\triangle$ CEA was utilized as a positive control to compare with $25(\mathrm{OH}) \mathrm{D}$ level in our study. There is a hypothesis that alteration of vitamin $D$ receptor may change transcriptional control of 1, 25(OH) $2 \mathrm{D} 3$ and impact anti-tumor effect of vitamin D [15]. These results suggest that vitamin $D$ plays a role in inhibiting tumorigenesis of CRC. Our results showed that both serologic levels of pre- and post-nCRT CEA in LARC patients were distinctly higher than the healthy controls (Table 2). We also found that pre-nCRT CEA levels was a risk factor for $\operatorname{LARC}(\mathrm{OR}=13.145, \mathrm{Cl}=1.71-110.31, \mathrm{P}=0.012$, Table 3 , respectively $)$.

In our research, the serum concentration of pre-nCRT CEA was much lower than the post-nCRT level ( Table 2), while the concentration of pre-nCRT 25(OH)D was much higher than the post-nCRT level ( $P$ $<.001$, Table 2). It seems that CEA and 25(OH) D had an inverse association with the disease progression. However, there was no significant correlation between these two markers in the serum by Pearson's correlation analysis (Table 4) in our study. Perhaps the small sample volumes reduce the relationship between CEA and 25(OH) D. Due to the limitations of patient management in our hospital; we were unable to measure the serum CEA and $25(\mathrm{OH}) \mathrm{D}$ levels at multiple time points in this study.

Many studies found that there is an inverse association between serum 25(OH) D and the risk of CRC [16-18]. We found that both serologic levels of pre- and post- nCRT 25(OH) D in patients with LARC were significantly lower than in healthy individuals. Our research of univariate analysis showed that the serum concentration of pre- and post-nCRT 25(OH) D in male patients and tumor size $<5.0 \mathrm{~cm}$ were significantly higher (Table1). A potential explanation could be that a more advanced stage of disease and/or larger tumor size is accompanied with more inflammation, which may lead to lower 25(OH) D3 levels [19, 20]. It could be that the absorption of vitamin $\mathrm{D}$ from diet and supplements is impaired due to subclinical 
mucositis induced by chemotherapy [21,22]. Andersen et al found that the inverse association between serum 25(OH) D and CRC was more prominent in females than males [6]. A meta-analysis including 1248 cases of $\mathrm{CRC}$ suggested that $25(\mathrm{OH}) \mathrm{D}$ had stronger inverse associations with colon than with rectal tumors [23]. However, in our study, the serum concentration of pre- and post-nCRT 25(OH) D in rectal and colon tumors were not statistically significant. The serum concentration of pre- and post-nCRT CEA in groups of tumor size $\$ 5.0 \mathrm{~cm}$ and lymph node invasion patients not significantly reduced.

A report suggests that low vitamin D levels were found in most cases of breast cancer with advanced tumor stage, large tumors, and positive lymph nodes[24]. In line with our results, the study of Fakih and colleagues observed a threefold higher risk of having vitamin D deficiency in CRC patients[25]. In line with our results, the study of Isenring and colleagues also reported a decrease in 25(OH) D levels in cancer patients receiving chemotherapy [26]. The lower 25(OH)D3 levels and/or the steeper decrease in 25(OH)D3 levels in patients receiving chemotherapy can be induced by changes in lifestyle due to chemotherapy or by chemotherapy directly[27]. The study of Churilla et al., found a higher prevalence of vitamin D deficiency in stage III of disease[28]. But, Some studies show that no difference in the 25(OH) D levels from baseline to six-month follow-up between patients who did and did not receive chemotherapy $(P=0.26)$, suggesting that chemotherapy may not have the deleterious effects on 25(OH)D levels that we ascribed to increased photosensitivity and potential for gastrointestinal toxicities [29]. And furthermore, Song et al. have found that the associations between vitamin D deficiency and CRC in a large nested case-control study remain valid after adjustment for inflammatory markers [30].

\section{Conclusions}

In conclusion, our study found that there was a relationship between serum 25(OH) D and CEA in LARC. Thus, serum 25(OH) D and CEA levels might be of value in determining the pathogenesis and risk of LARC and requires further study.

\section{Declarations}

\section{Ethics approval and consent to participate}

The study was conducted according to the guidelines laid down in the Declaration of Helsinki and was approved by the Institutional Review Board of the Changzhou Cancer Hospital Autonomous Region for the use of human materials. All participants provided informed consent prior to participation.

\section{Consent for publication}

All authors read and approved the final manuscript.

\section{Availability of data and materials}


The datasets during and/or analyzed during the current study are available from the corresponding author on reasonable request.

\section{Competing interests}

The authors declare that they have no competing of interest.

\section{Funding}

This study was supported by the Changzhou Cancer Hospital.

\section{Authors' contributions}

All authors contributed to the study conception and design and take responsibility for the integrity of the

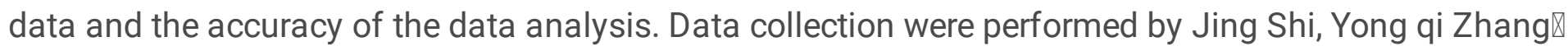
Hong Ye, Xiang He, and analysis were performed by Litao Yu. Material preparation and the first draft of the manuscript were written by Litao Yu. And all authors commented on previous versions of the manuscript. All authors read and approved the final manuscript.

\section{Acknowledgments}

We are grateful to the participants, interviewers, and researchers from the Changzhou Cancer Hospital.

\section{Author details}

1. Changzhou Cancer Hospital Affiliated to Soochow University, Changzhou, Jiangsu, China

2. Changzhou Maternal And Child Health Care Hospital, Changzhou, Jiangsu, China

\section{References}

1. Torre, L.A., et al., Global cancer statistics, 2012. CA Cancer J Clin, 2015. 65(2): p. 87-108.

2. Chen, W., et al., Cancer statistics in China, 2015. CA Cancer J Clin, 2016. 66(2): p. 115-32.

3. Benson, A.B., 3rd, et al., Anal Carcinoma, Version 2.2012: featured updates to the NCCN guidelines. J Natl Compr Canc Netw, 2012. 10(4): p. 449-54.

4. Song, M., et al., Plasma 25-hydroxyvitamin D and colorectal cancer risk according to tumour immunity status. Gut, 2016. 65(2): p. 296-304.

5. Printz, C., High vitamin D levels increase survival rates in patients with metastatic colorectal cancer. Cancer, 2015. 121(13): p. 2105.

6. Andersen, S.W., et al., Total and Free Circulating Vitamin D and Vitamin D-Binding Protein in Relation to Colorectal Cancer Risk in a Prospective Study of African Americans. Cancer Epidemiol Biomarkers Prev, 2017. 26(8): p. 1242-1247. 
7. Peterlik, M. and H.S. Cross, Vitamin D and calcium insufficiency-related chronic diseases: molecular and cellular pathophysiology. Eur J Clin Nutr, 2009. 63(12): p. 1377-86.

8. Aranow, C., Vitamin D and the immune system. J Investig Med, 2011. 59(6): p. 881-6.

9. Kim, C.H., et al., Prognostic Effect of Pretreatment Serum Carcinoembryonic Antigen Level: A Useful Tool for Prediction of Distant Metastasis in Locally Advanced Rectal Cancer Following Neoadjuvant Chemoradiotherapy and Total Mesorectal Excision. Medicine (Baltimore), 2015. 94(31): p. e1291.

10. Huh, J.W., et al., Accuracy of endorectal ultrasonography and computed tomography for restaging rectal cancer after preoperative chemoradiation. J Am Coll Surg, 2008. 207(1): p. 7-12.

11. Hur, H., et al., Can a biomarker-based scoring system predict pathologic complete response after preoperative chemoradiotherapy for rectal cancer? Dis Colon Rectum, 2014. 57(5): p. 592-601.

12. Moreno Garcia, V., et al., Prognostic value of carcinoembryonic antigen level in rectal cancer treated with neoadjuvant chemoradiotherapy. Int J Colorectal Dis, 2009. 24(7): p. 741-8.

13. Yang, K.L., et al., Carcinoembryonic antigen (CEA) level, CEA ratio, and treatment outcome of rectal cancer patients receiving pre-operative chemoradiation and surgery. Radiat Oncol, 2013. 8: p. 43.

14. Kim, C.W., et al., Clinical significance of pre- to post-chemoradiotherapy s-CEA reduction ratio in rectal cancer patients treated with preoperative chemoradiotherapy and curative resection. Ann Surg Oncol, 2011. 18(12): p. 3271-7.

15. Poynter, J.N., et al., Genetic variation in the vitamin D receptor (VDR) and the vitamin D-binding protein (GC) and risk for colorectal cancer: results from the Colon Cancer Family Registry. Cancer Epidemiol Biomarkers Prev, 2010. 19(2): p. 525-36.

16. Song, M., et al., Plasma 25-Hydroxyvitamin D, Vitamin D Binding Protein, and Risk of Colorectal Cancer in the Nurses' Health Study. Cancer Prev Res (Phila), 2016. 9(8): p. 664-72.

17. Chandler, P.D., et al., Circulating Vitamin D Levels and Risk of Colorectal Cancer in Women. Cancer Prev Res (Phila), 2015. 8(8): p. 675-82.

18. Atoum, M.F. and M.N. Tchoporyan, Association between circulating vitamin D, the Taq1 vitamin D receptor gene polymorphism and colorectal cancer risk among Jordanians. Asian Pac J Cancer Prev, 2014. 15(17): p. 7337-41.

19. Hanahan, D. and R.A. Weinberg, Hallmarks of cancer: the next generation. Cell, 2011. 144(5): p. 64674.

20. Mangin, M., R. Sinha, and K. Fincher, Inflammation and vitamin D: the infection connection. Inflamm Res, 2014. 63(10): p. 803-19.

21. Fakih, M.G., et al., Chemotherapy is linked to severe vitamin D deficiency in patients with colorectal cancer. Int J Colorectal Dis, 2009. 24(2): p. 219-24.

22. Peterson, D.E., et al., Management of oral and gastrointestinal mucositis: ESMO Clinical Practice Guidelines. Ann Oncol, 2011. 22 Suppl 6: p. vi78-84.

23. Jenab, M., et al., Association between pre-diagnostic circulating vitamin D concentration and risk of colorectal cancer in European populations:a nested case-control study. BMJ, 2010. 340: p. b5500. 
24. Thanasitthichai, S., A. Chaiwerawattana, and A. Prasitthipayong, Association of Vitamin D Level with Clinicopathological Features in Breast Cancer. Asian Pac J Cancer Prev, 2015. 16(12): p. 4881-3.

25. Toriola, A.T., et al., Circulating 25-hydroxyvitamin D levels and prognosis among cancer patients: a systematic review. Cancer Epidemiol Biomarkers Prev, 2014. 23(6): p. 917-33.

26. Isenring, E.A., et al., Serum vitamin D decreases during chemotherapy: an Australian prospective cohort study. Asia Pac J Clin Nutr, 2018. 27(5): p. 962-967.

27. Wesselink, E., et al., Chemotherapy and vitamin D supplement use are determinants of serum 25hydroxyvitamin D levels during the first six months after colorectal cancer diagnosis. J Steroid Biochem Mol Biol, 2020. 199: p. 105577.

28. Churilla, T.M., et al., Vitamin D deficiency is widespread in cancer patients and correlates with advanced stage disease: a community oncology experience. Nutr Cancer, 2012. 64(4): p. 521-5.

29. Savoie, M.B., et al., Vitamin D Levels in Patients with Colorectal Cancer Before and After Treatment Initiation. J Gastrointest Cancer, 2019. 50(4): p. 769-779.

30. Song, M., et al., Plasma 25-hydroxyvitamin D and risk of colorectal cancer after adjusting for inflammatory markers. Cancer Epidemiol Biomarkers Prev, 2014. 23(10): p. 2175-80. 